\title{
Study of HTS Wires at High Magnetic Fields
}

\author{
D. Turrioni, E. Barzi, M. J. Lamm, R. Yamada, A. V. Zlobin, A. Kikuchi
}

\begin{abstract}
Fermilab is working on the development of high field magnet systems for ionization cooling of muon beams. The use of high temperature superconducting (HTS) materials is being considered for these magnets using Helium refrigeration. Critical current $\left(I_{c}\right)$ measurements of HTS conductors were performed at FNAL and at NIMS up to $28 \mathrm{~T}$ under magnetic fields at zero to 90 degree with respect to the sample face. A description of the test setups and results on a BSCCO-2223 tape and second generation (2G) coated conductors are presented.
\end{abstract}

Index Terms - High temperature superconductor, BSCCO, coated conductor, critical current

\section{INTRODUCTION}

$\mathrm{T}_{\mathrm{s}}^{\mathrm{H}}$ HE main application of High Temperature Superconductor (HTS) magnets at Fermilab is presently in the realm of Muon Colliders, which require high field solenoids for muon beam cooling. This includes the high field sections of a 6D Helical Cooling Channel [1,2] and high-field solenoids $(>30 \mathrm{~T})$ for the final, low emittance stage of the muon cooling channel [3-5]. These ambitious goals require strong HTS magnet and conductor programs, to be conducted in close collaboration with other U.S. National Labs, Universities and Industry.

A main mission of the conductor program is that of monitoring and studying the best performing HTS's on the market, including state-of-the-art Second Generation (2G) coated conductors, but also Bi-2212 round wire and cables [6] together with the U. S. National Collaboration. Characterizing HTS materials, the engineering current density $\left(J_{\mathrm{E}}\right)$ as a function of magnetic field, temperature, field orientation, as well as transverse, longitudinal and bending strain/stress is essential input to practical magnet design. To monitor Industry progress, HTS tapes need extensive evaluation of the aforementioned properties. Based on conductor results, the best performing HTS conductors will be selected to be used in the magnet program.

BSCCO-2223 and 2 G HTS are typically produced in the form of tapes, which are anisotropic and exhibit the highest critical current when the magnetic field is applied parallel to the tape face $\left(\mathrm{B}_{\mathrm{PAR}}\right)$ and the lowest one when the field is perpendicular to it $\left(\mathrm{B}_{\mathrm{PERP}}\right)$. Fig. 1 shows the geometrical configuration of a tape and relative directions of magnetic

Manuscript received August 26, 2008.

This work was supported by the U.S. Department of Energy.

D. Turrioni, E. Barzi, M. J. Lamm, R. Yamada, A. V. Zlobin are with the Fermi National Accelerator Laboratory (Fermilab), P.O. Box 500, Batavia, IL 60510 USA (phone: 630-840-3695; fax: 630-840-3369; e-mail: turrioni@fnal.gov). A. Kikuchi is with the National Institute of Materials Science Tsukuba, Ibaraki, 3050048, Japan. field. Earlier $1 \mathrm{G}$ tapes have been tested at various temperatures in both field directions [7,8]. Angular measurements on $2 \mathrm{G}$ have been performed at higher temperatures $[9,10]$.

A sample holder designed to perform $I_{c}$ measurements of HTS tapes under externally applied magnetic fields, with orientations $\Theta$ varying from zero to 90 degrees with respect to the c-axis (see Fig. 1), was used in this study. The $I_{c}$ angular dependence was measured at FNAL at temperatures from $4.2 \mathrm{~K}$ to $33 \mathrm{~K}$ up to $15 \mathrm{~T}$, while providing up to $1400 \mathrm{~A}$ of current to a $2 \mathrm{G}$ sample by SuperPower (SP). To complement similar studies on two HTS conductors by American Superconductor (AMSC) [11], the latter plus a sample by SP were tested at $4.2 \mathrm{~K}$ in parallel and perpendicular field configurations up to $28 \mathrm{~T}$ at NIMS.

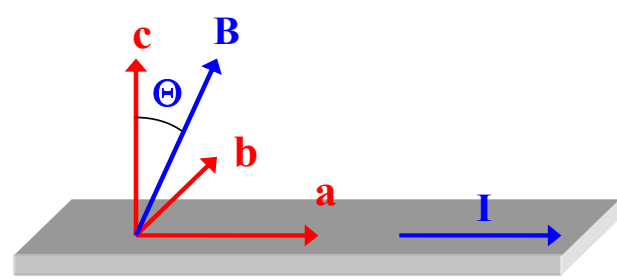

Fig. 1. HTS superconductor with current direction and relative direction of magnetic field B (Schematic is courtesy of C. Thieme, AMSC).

\section{SAMPLES DESCRIPTION}

First Generation (1G) multi-filamentary HTS are composites of silver or silver alloy matrix and BSCCO [12]. $2 \mathrm{G}$ HTS conductor is based on a thin film approach, and it is now seen as a major candidate for an effective replacement of $1 \mathrm{G}$ wire. $2 \mathrm{G}$ has excellent mechanical properties, and allows a react-and-wind technique for magnet construction.

TABLE I HTS SPECIFICATIONS

\begin{tabular}{lccc}
\hline \hline Strand ID & Hermetic 1G & 2G 348 & 2G SCS 4050 \\
Company & AMSC & AMSC & SuperPower \\
Min $\mathrm{I}_{\mathrm{c}}$ (77 K, self-field) & 115 & 110 & 100 \\
Average thickness $\mathrm{t}_{\mathrm{T}}$ & $0.31 \mathrm{~mm}$ & $0.2 \mathrm{~mm}$ & $0.143 \mathrm{~mm}$ \\
Average width $\mathrm{w}_{\mathrm{T}}$ & $4.8 \mathrm{~mm}$ & $4.8 \mathrm{~mm}$ & $4.33 \mathrm{~mm}$ \\
Laminate & stainless & copper & copper \\
Laminate thickness & $2 \times 0.037 \mathrm{~mm}$ & $2 \times 0.050 \mathrm{~mm}$ & $2 \times 0.020 \mathrm{~mm}$ \\
YBCO layer thickness & N.A. & $1.4 \mu \mathrm{m}$ & $1.2 \mu \mathrm{m}$ \\
Min. critical bend diameter & $50 \mathrm{~mm}$ & $50 \mathrm{~mm}$ & $20 \mathrm{~mm}$ \\
Max. rated tensile strain & $0.3 \%$ & $0.3 \%$ & $0.45 \%$ \\
\hline \hline
\end{tabular}

A BSCCO-2212 round wire by Oxford Superconducting Technology (OST) [6], a BSCCO-2223 Hermetic tape and a $2 \mathrm{G} 348$ Coated conductor by AMSC, as well as a $2 \mathrm{G}$ SCS 4050 conductor by SP were used in this study. The tape specifications are given in Table I. A schematic of the SP 
conductor is shown in Fig. 2. Pictures of the AMSC conductors can be found in [11].

For both $2 \mathrm{G}$ conductors, the active component is the $\mathrm{YBCO}$ $\left(\mathrm{YBa}_{2} \mathrm{Cu}_{3} \mathrm{O}_{7}\right)$, which is only about $1 \mu \mathrm{m}$ thick. AMSC uses a bi-axially textured substrate approach onto which a thin epitaxial oxide buffer layer is deposited $\left(\operatorname{RABiTS}^{\mathrm{TM}}\right)[13,14]$. SP uses the IBAD technique $[15,16]$.

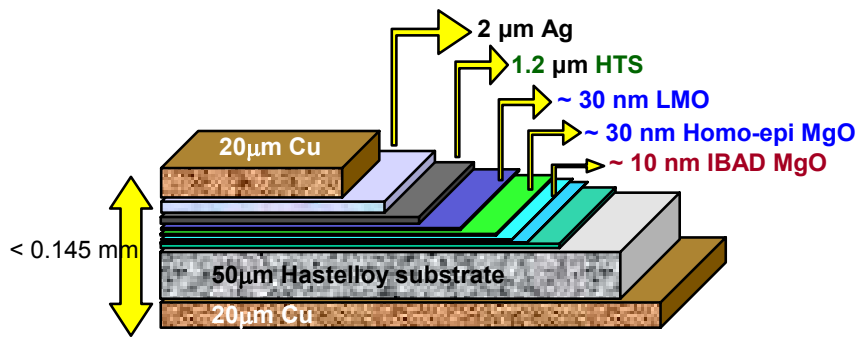

Fig. 2. Schematic of a $2 \mathrm{G}$ coated conductor by SuperPower showing an YBCO layer $1.2 \mu \mathrm{m}$ thick obtained with the IBAD technique.

\section{EXPERIMENTAL SETUPS}

\section{A. Measurement Procedure at FNAL}

The samples used at FNAL were straight and $38 \mathrm{~mm}$ long. The sample, supported in its middle part by G-10, was soldered within a groove on two $\mathrm{Cu}$ half cylinders using a splice length of $12 \mathrm{~mm}$ at each sample end to control contact resistance and heating power. The sample holder was placed at the desired angle within cylindrical holes in the probe $\mathrm{Cu}$ lugs [11]. The distance between the voltage taps was of $10 \mathrm{~mm}$.

At FNAL measurements were obtained at a number of temperatures in a Variable Temperature Insert (VTI) with an inner diameter of $49 \mathrm{~mm}$, within a $15 / 17 \mathrm{~T}$ magneto-cryostat and 2000 A current leads to the sample. The temperature was controlled using a LabView PID routine and a LakeShore218 monitor to read the Cernox ${ }^{\mathrm{TM}}$ sensor on the sample. The average temperatures at which the measurements were performed in parallel field were $4.234 \mathrm{~K} \pm 0.019 \mathrm{~K}, 14.113 \mathrm{~K}$ $\pm 0.037 \mathrm{~K}, 22.212 \mathrm{~K} \pm 0.134 \mathrm{~K}$ and $33.082 \mathrm{~K} \pm 0.074 \mathrm{~K}$. The average temperature variation during the superconducting to normal transition was negligible at $4.2 \mathrm{~K}, 0.126 \mathrm{~K}$ at 14.113 $\mathrm{K}, 0.097 \mathrm{~K}$ at $22.212 \mathrm{~K}$, and $0.116 \mathrm{~K}$ at $33.082 \mathrm{~K}$. For the angular dependence measurements, the angle $\Theta$ was changed in steps of 11.250 and 22.50 degrees. First, critical current measurements were performed in liquid nitrogen $(77 \mathrm{~K})$ at self-field. Next, voltage-current (VI) characteristics were measured in $\mathrm{He}$ (liquid or vapor) at a magnetic field between 0 and $15 \mathrm{~T}$. The critical current $\mathrm{I}_{\mathrm{c}}$ was determined using the $1 \mu \mathrm{V} / \mathrm{cm}$ criterion [17]. Critical currents have not been corrected for self-field effects which can be substantial at low fields.

\section{B. Measurement Procedure at NIMS}

The sample holder used at NIMS is shown in Fig. 3. Six samples were tested in series. Three were mounted in a parallel field configuration and three in a perpendicular field configuration. Samples were straight and $30 \mathrm{~mm}$ long. They were supported on G-10 $5 \mathrm{~mm}$ wide holders ending with $\mathrm{Cu}$ caps. Splice length was $5 \mathrm{~mm}$ at each sample end. The distance between the voltage taps was $5 \mathrm{~mm}$. The critical current $I_{c}$ was determined using the $1 \mu \mathrm{V} / \mathrm{cm}$ criterion. At NIMS the VI characteristics were measured in liquid $\mathrm{He}$ at magnetic fields up to $28 \mathrm{~T}$ in the $30 \mathrm{~T}$ Hybrid magnet with a $52 \mathrm{~mm}$ warm bore. The equipment power supply limit was $500 \mathrm{~A}$.

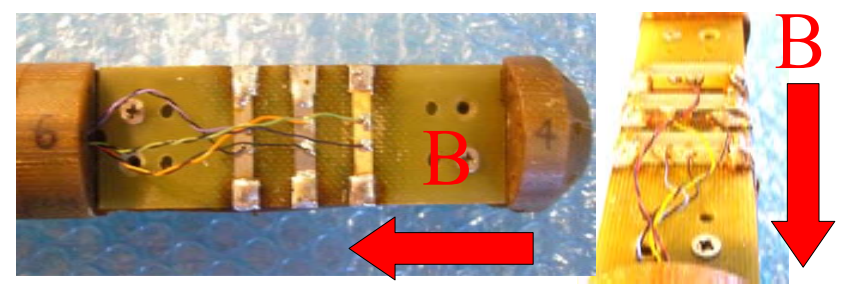

Fig. 3. Sample holder used at NIMS showing six instrumented samples.

\section{RESUlTS}

\section{A. Critical Current Data}

Fig. 4 shows $I_{c}$ results at $4.2 \mathrm{~K}$ for the HTS wires as a function of magnetic field as measured at NIMS and at FNAL [11]. For the tapes both the parallel and perpendicular field configurations were used. There is a good agreement for the $\mathrm{BSCCO}$ round wire and the $2 \mathrm{G}$ conductors, whereas some discrepancies are apparent for the $1 \mathrm{G}$ hermetic tape. This is due to the hysteretic $\mathrm{I}_{\mathrm{c}}(\mathrm{B})$ behavior [18] of BSCCO-2223 when tested in increasing (FNAL) and decreasing (NIMS) magnetic field.

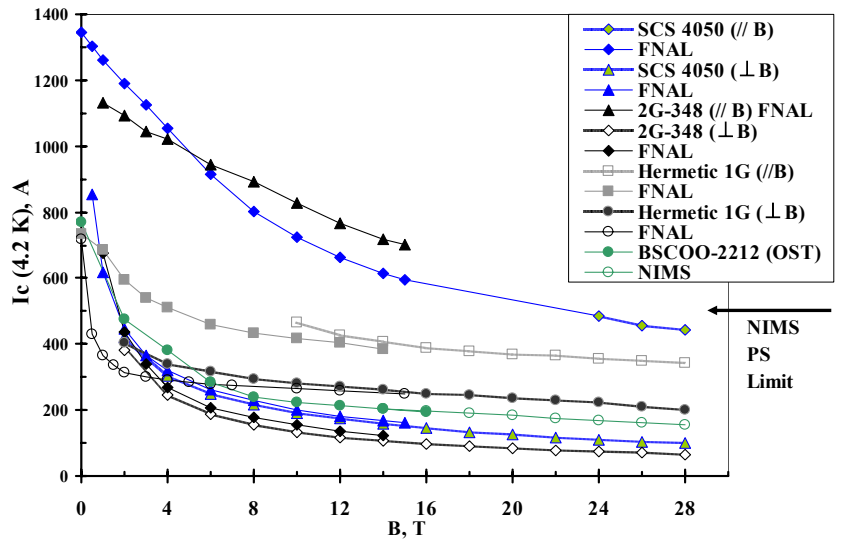

Fig. 4. $\mathrm{I}_{\mathrm{c}}(\mathrm{B})$ results at $4.2 \mathrm{~K}$ of the HTS wires obtained at NIMS, compared with FNAL results [11]. The tapes were tested both in parallel and perpendicular fields.

\section{B. Measurement of Anisotropy}

Fig. 5 shows $I_{c}$ results as a function of magnetic field for the 2G SCS 4050 conductor, in the parallel and perpendicular field configurations, from $4.2 \mathrm{~K}$ to $33 \mathrm{~K}$. The performance at self-field and $77 \mathrm{~K}$ was $106 \pm 1 \mathrm{~A}$. One can see that the $\mathrm{I}_{\mathrm{c}}$ dependence in parallel field reduces mostly linearly with field, which indicates that effective pinning is maintained for the parallel direction over the entire field range. For the 
perpendicular direction pinning is most effective at low fields but then reduces at high fields.

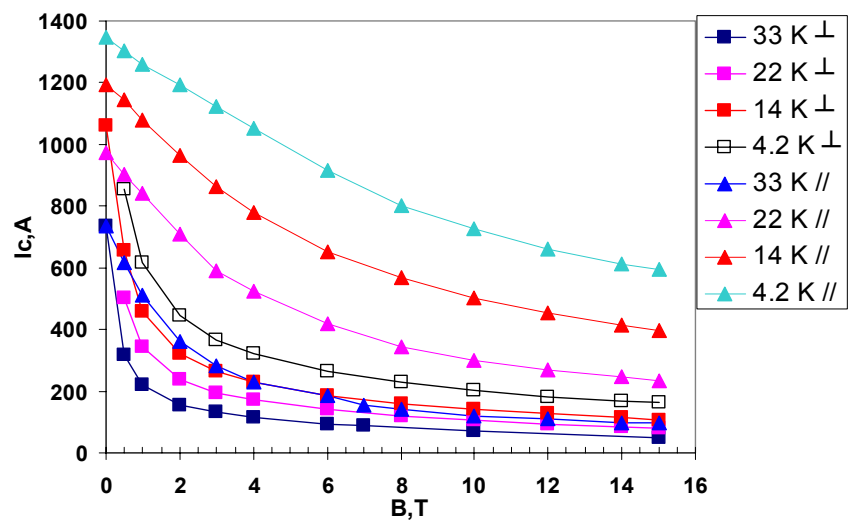

Fig. 5. $I_{c}(B, T)$ of the $2 \mathrm{G}$ SCS 4050 coated conductor in parallel and perpendicular fields, from $4.2 \mathrm{~K}$ to $33 \mathrm{~K}$.

Figs. 6 and 7 show the $I_{c}$ normalized to $I_{c}(77 \mathrm{~K}, 0 \mathrm{~T})$ as a function of field and field angle at $4.2 \mathrm{~K}$ and $33 \mathrm{~K}$. At $4.2 \mathrm{~K}$ the peak in $\mathrm{I}_{\mathrm{c}}$ is at $90^{\circ}$ whereas at $33 \mathrm{~K}$ it is shifted by a few degrees. At $4.2 \mathrm{~K}$ the $\mathrm{I}_{\mathrm{c}}$ sensitivity to $\Theta$ increases at increasing fields, but it is always lower than at $33 \mathrm{~K}$.

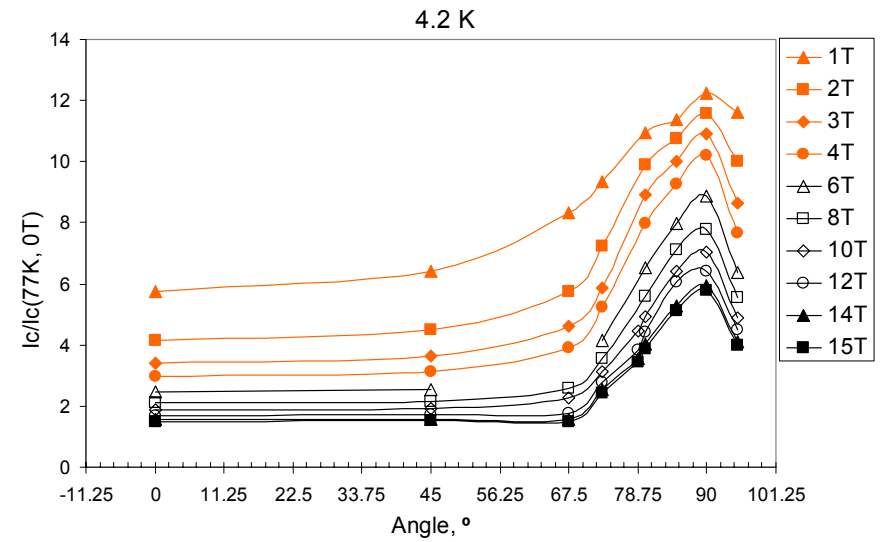

Fig. 6. Normalized $\mathrm{I}_{\mathrm{c}}(4.2 \mathrm{~K})$ of the $2 \mathrm{G} \mathrm{SCS} 4050$ coated conductor versus B and $\Theta$.

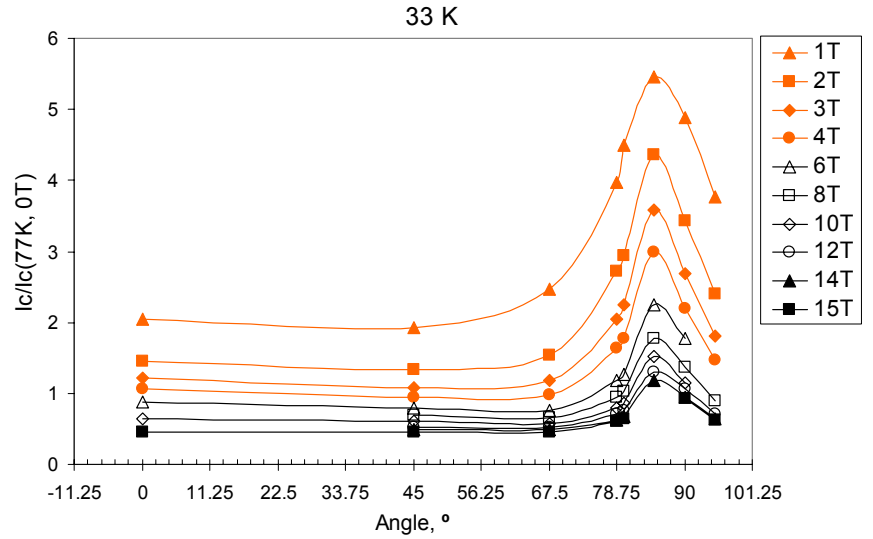

Fig. 7. Normalized $\mathrm{I}_{\mathrm{c}}(33 \mathrm{~K})$ of the $2 \mathrm{G}$ SCS 4050 coated conductor versus $B$ and $\Theta$.
To gauge field and temperature dependence of the anisotropy, the ratio of $I_{c}$, normalized to $I_{c}(77 \mathrm{~K}, 0 \mathrm{~T})$, in parallel field to that in perpendicular field as a function of applied magnetic field, or:

$$
R_{I_{C}}=\frac{I_{C}\left(B_{P A R}\right) / I_{C}(77 K, 0 T)_{P A R}}{I_{C}\left(B_{P E R P}\right) / I_{C}(77 K, 0 T)_{P E R P}}
$$

can be calculated at the various temperatures and fields. The subscripts $P A R$ and PERP for $\mathrm{I}_{\mathrm{c}}(77 \mathrm{~K}, 0 \mathrm{~T})$ indicate the $\mathrm{I}_{\mathrm{c}}(77$ $\mathrm{K}, 0 \mathrm{~T}$ ) of the samples used for the parallel and perpendicular tests. This ratio is plotted in Figs. 8 and 9 for the 2G SCS 4050 and the $1 \mathrm{G}$ hermetic tapes, using for data at $4.2 \mathrm{~K}$ both FNAL and NIMS results. For the $1 \mathrm{G}$ tape, a linear field dependence with a slope increasing with temperature had been produced for this ratio [11]. The NIMS data show a reasonably flat field dependence at $4.2 \mathrm{~K}$ (Fig. 9), making it a good choice at 4.2 K. For the 2G SCS 4050 conductor (Fig. 8), the ratio increases fast with field and tends to saturate above $4 \mathrm{~T}$. The NIMS data show that at $4.2 \mathrm{~K}$ it actually slightly increases at high field. Contrary to the $2 \mathrm{G} 348$ conductor, where this ratio showed no observable temperature dependence [11], in the case of the $2 \mathrm{G}$ SCS 4050, above $4 \mathrm{~T}$ the ratio decreases with increasing temperatures. This behavior at the higher temperatures is caused also by the shift of the peak current to field angles that are not parallel to the tape, as for instance observed at $33 \mathrm{~K}$ in Fig. 7.

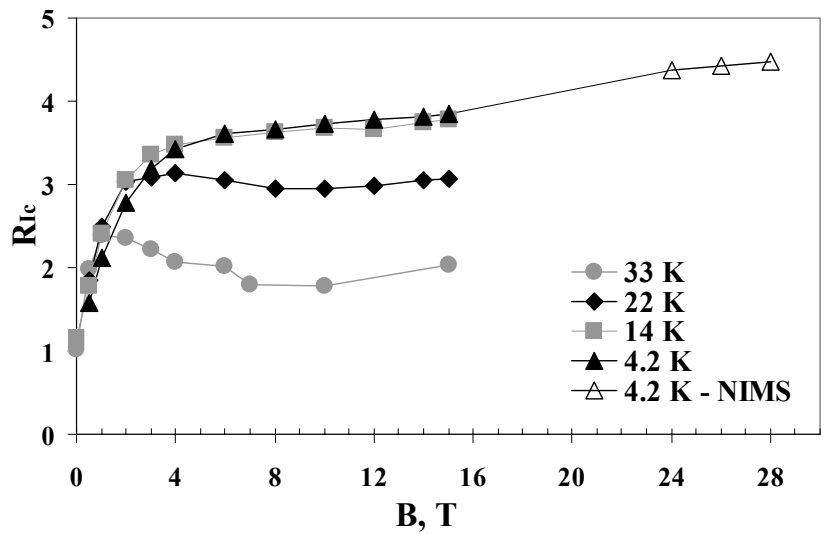

Fig. 8. Ratio of normalized $\mathrm{I}_{\mathrm{c}}$ in parallel and perpendicular fields, from $4.2 \mathrm{~K}$ to $33 \mathrm{~K}$, for the $2 \mathrm{G} \mathrm{SCS} 4050$ tape.

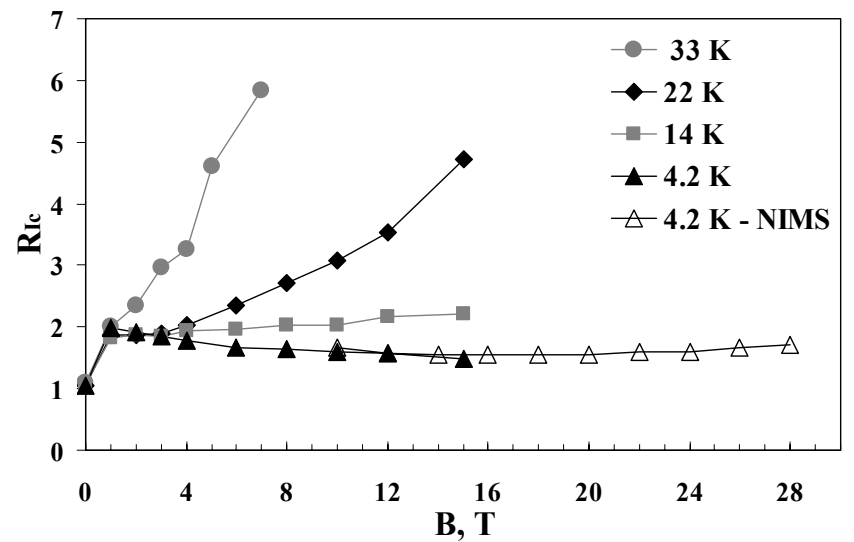

Fig. 9. Ratio of normalized $I_{c}$ in parallel and perpendicular fields, from $4.2 \mathrm{~K}$ to $33 \mathrm{~K}$ [11], for the $1 \mathrm{G}$ hermetic tape. 


\section{Comparison with LTS}

Fig. 10 shows the engineering critical current $\mathrm{J}_{\mathrm{E}}$ at $4.2 \mathrm{~K}$ and magnetic fields up to $28 \mathrm{~T}$ for the HTS wires, compared with that of $\mathrm{Nb}_{3} \mathrm{Sn}$ and $\mathrm{NbTi}$ wires. For the calculation of $\mathrm{J}_{\mathrm{E}}$ the entire cross section of each conductor was used. Below 14 to $15 \mathrm{~T}$, the $\mathrm{J}_{\mathrm{E}}$ of the RRP $\mathrm{Nb}_{3} \mathrm{Sn}$ wire exceeds that of all HTS at $4.2 \mathrm{~K}$. Above $17 \mathrm{~T}$ the Bi-2212 round conductor shows a good overall performance. The $2 \mathrm{G}$ superconductors have a much higher current density in parallel fields, indicating excellent potential for high current density insert magnets for very high fields. For complete solenoids in which end sections operate with high perpendicular field components, the $\mathrm{B}_{\text {PERP }}$ performance is currently too low and needs further pinning enhancement for this field direction. It is interesting that the Bi-2223 conductor, which at lower fields has a lower $J_{\mathrm{E}}$ than other HTS's, actually recovers at the highest fields.

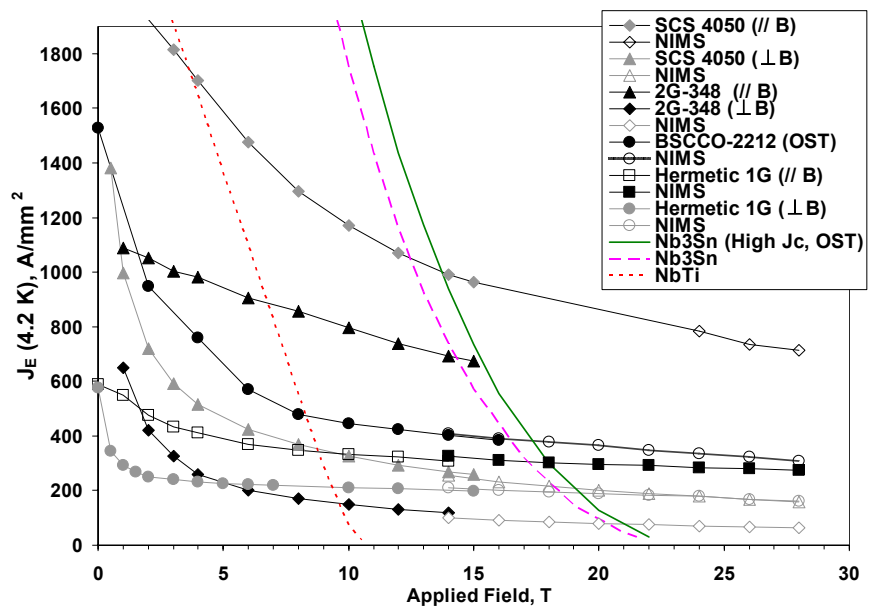

Fig. 10. $\mathrm{J}_{\mathrm{E}}(\mathrm{B})$ at $4.2 \mathrm{~K}$ for HTS coated conductors, Bi-2223 tape, and Bi-2212, $\mathrm{Nb}_{3} \mathrm{Sn}$ and $\mathrm{NbTi}$ round wires [11].

\section{CONCLUSION}

The $I_{c}$ angular dependence on field orientation was measured at FNAL at temperatures from $4.2 \mathrm{~K}$ to $33 \mathrm{~K}$ up to $15 \mathrm{~T}$, while providing up to $1400 \mathrm{~A}$ of current to a $2 \mathrm{G}$ sample by SuperPower (SP). To complement similar studies $[6,11]$, the latter plus a BSCCO-2212 round wire by OST, and a BSCCO-2223 Hermetic tape and a 2G 348 Coated conductor by AMSC were tested at $4.2 \mathrm{~K}$ up to $28 \mathrm{~T}$ at NIMS. For the tapes both the parallel and perpendicular field configurations were used.

The low anisotropy and reasonably flat field dependence at $4.2 \mathrm{~K}$ of the $1 \mathrm{G}$ hermetic wire makes it a good choice at $4.2 \mathrm{~K}$. On the other hand, for the $2 \mathrm{G} \mathrm{SCS} 4050$ conductor at $4.2 \mathrm{~K}$ the peak in $\mathrm{I}_{\mathrm{c}}$ is at $90^{\circ}$, whereas at $33 \mathrm{~K}$ it is shifted by a few degrees. Also, at $4.2 \mathrm{~K}$ the $I_{c}$ sensitivity to $\Theta$ increases at increasing fields, but it is always lower than at $33 \mathrm{~K}$.

The $\mathrm{J}_{\mathrm{E}}$ in parallel magnetic field of state-of-the-art coated conductors has dramatically increased in recent years. However, solenoids with strong perpendicular field components in the end sections are limited by the critical current angular sensitivity. Reducing conductor anisotropy while preserving at the same time the peak $\mathrm{J}_{\mathrm{E}}$ would considerably increase the performance of these magnets.

\section{ACKNOWLEDGMENT}

The authors wish to thank SuperPower, Inc. and American Superconductor Corporation for kindly providing the HTS wire samples for this work.

\section{REFERENCES}

[1] S. A. Kahn et al., "Magnet Systems for Helical Muon Cooling Channels", Proceedings of PAC07, Albuquerque, NM, June 2007.

[2] V. V. Kashikhin et al., "Design Studies of Magnet Systems For Muon Helical Cooling Channels", EPAC2008, Genoa, Italy, June 2008.

[3] S. A. Kahn et al., "High Field Solenoid Magnets for Muon Cooling", Proceedings of EPAC2006, Edinburgh, Scotland, pp. 2634-2536.

[4] S. A. Kahn et al., "A High Field HTS Solenoid for Muon Cooling", Proceedings of PAC07, Albuquerque, NM, June 2007.

[5] V. V. Kashikhin et al., "Study of High Field Superconducting Solenoids for Muon Beam Cooling", IEEE Trans. Appl. Sup., V. 18, No. 2, pp. 928-932 (2007).

[6] E. Barzi et al., "BSCCO-2212 Wire and Cable Studies", Advances in Cryogenic Engineering, AIP, V. 54, p. 431 (2008).

[7] M. P. Maley, J. H. Cho, J. Y. Coulter, J. O. Willis, L. N. Bulaevskii, L. R. Motowidlo, and P. Haldar, "Anisotropy of transport properties normal and parallel to the tape plane in Bi-2223/Ag tapes," IEEE Trans. Appl. Sup., V. 5, pp. 1290-1293 (1995).

[8] B. Xu, J. H. Su and J. Schwartz, "Dependence of transport critical current of magnetic field processed $\mathrm{Bi}_{2} \mathrm{Sr}_{2} \mathrm{CaCu}_{2} \mathrm{O}_{\mathrm{x}} / \mathrm{AgMg}$ tapes on the background magnetic field and magnetic field direction," Superconductor Science and Technology, 18, pp. 503-507 (2005).

[9] T. Holesinger and L. Civale," Multi-scale Characterization of Structure and Properties of Coated Conductors", presented at the U.S. DOE Superconductivity for Electric Systems Annual Peer Review, August 2006, Washington, D.C.

[10] "Progress in Scale-up of 2G HTS Wire at SuperPower," presented at the U.S. DOE Superconductivity for Electric Systems Annual Peer Review, July 29-31, 2008, Arlington, VA.

[11] D. Turrioni et al., "Angular Measurements of HTS Critical Current for High Field Solenoids", Advances in Cryogenic Engineering, AIP, V. 54, p. 451 (2008).

[12] W. Zhang, Y. Huang, X. Li, T. Kodenkandath, M.W. Rupich, U. Schoop, D. T.Verebelyi, C.L.H. Thieme, E. Siegal, T. G. Holesinger, B. Maiorov, L. Civale, D. J. Miller, V. A. Maroni, J. Li, P. M. Martin, E. D. Specht, A. Goyal, and M. P. Paranthaman, "Control of Flux Pinning in MOD YBCO Coated Conductor”, IEEE Trans. Appl. Sup., V. 17, No. 2, p. 3347 (2007).

[13] M. W. Rupich, U. Schoop, C. Thieme, D. T. Verebelyi, W. Zhang, X. Li, T. Kodenkandath, N. Nguyen, E. Siegal, L. Civale, T. Holesinger, A. Goyal, and M. Paranthaman,"Second Generation HTS Wire Based on RABiTS $^{\mathrm{TM}}$ Substrates and MOD YBCO", IEEE Trans. Appl. Sup., V. 15 , p. 2458 (2005).

[14] M. W. Rupich, U. Schoop, D. T. Verebelyi, C. L. H. Thieme, D. Buczek, X. Li, W. Zhang, T. Kodenkandath, Y. Huang, E. Siegal, W. Carter, N. Nguyen, J. Schreiber, M. Prasova, J. Lynch, D. Tucker, R. Harnois, C. King, and D. Aized, "The Development of Second Generation HTS Wire at American Superconductor", IEEE Trans. Appl. Sup., V. 17, No. 2, p. 3379 (2007).

[15] X. Xiong, K. P. Lenseth, J. L. Reeves, A. Rar, Y. Qiao, R. M. Schmidt, Y. Chen, Y. Li, Y. Y. Xie, and V. Selvamanickam, "High Throughput Processing of Long-Length IBAD MgO and Epi-Buffer Templates at SuperPower", IEEE Trans. Appl. Sup., V. 17, No. 2, p. 3375 (2007).

[16] V. Selvamanickam, Y. Chen, X. Xiong, Y. Y. Xie, J. L. Reeve, X. Zhang, Y. Qiao, K. P. Lenseth, R. M. Schmidt, A. Rar, D. W. Hazelton, and K. Tekletsadik, "Recent Progress in Second-Generation HTS Conductor Scale-Up at SuperPower", IEEE Trans. Appl. Sup., V. 17, No. 2, p. 3231 (2007).

[17] E. Barzi et al., "High Temperature Superconductors for High Field Superconducting Magnets", Advances in Cryogenic Engineering, AIP, V. 52 , p. 416 (2006).

[18] J. E. Evetts and B. A. Glowacky, "Relation of critical current irreversibility to trapped flux and microstructure in polycrystalline YBCO”, Cryogenics, Vol. 28, pp. 641-649 (1988). 